\title{
INFANTILE MUSCULAR ATROPHY OF SPINAL ORIGIN
}

\section{A REPORT OF TWO CASES}

BY

J. G. MACLEOD, M.B., Ch.B., M.R.C.P.Ed., and

R. M. MACDONALD, B.Sc., M.B., Ch.B.

Two cases of infantile muscular atrophy of spinal origin were admitted in the summer of 1940 to the wards to which we were attached in the Royal Hospital for Sick Children, Edinburgh. They are considered of sufficient interest to be published in detail.

\section{Review and discussion of literature}

In 1891 Werdnig described cases of muscular atrophy in infants characterized by wasting and paralysis of the muscles of the trunk, neck and proximal limb segments, beginning about the tenth month of life. Tendon reflexes were lost. Bulbar symptoms, fibrillary twitchings and secondary contractures were present in some cases. There was no impairment of sensation or of mental development. Other children of the same family were affected. The disease ran a rapidly fatal course, and at autopsy the anterior horn cells were found to be atrophied, and secondary simple atrophy of the paralysed muscles was present. In 1893 Hoffmann confirmed Werdnig's description, and since then cases of this type have been known as examples of Werdnig-Hoffmann's disease. In 1900 Oppenheim described a syndrome characterized by a loss of power in all the muscles of the body. The muscles were small, hypotonia was marked, and contortionist attitudes possible. There were no localized atrophic areas as in the Werdnig-Hoffmann disease. Oppenheim's cases were further distinguished by being present from birth, by the absence of any familial involvement, and by a tendency to improve. Cases of this type are called Oppenheim's disease, amyotonia congenita, or myatonia congenita. Numerous other cases have since been described and much discussion has arisen about the features differentiating the two diseases. It has been found extremely difficult to determine constant distinguishing features.

Many different authorities, including Huenekens and Bell (1920), Greenfield and Stern (1927), Paterson (1928), and Tuthill and Levy (1931) have come to the conclusion that Werdnig-Hoffmann's and Oppenheim's diseases have an identical pathological basis, and that no sharp line of differentiation is possible clinically. The two probably represent extreme types of a disease in which many variations are possible.

It would appear undesirable therefore to continue to attempt to divide cases of muscular atrophy in infancy into cases of Werdnig-Hoffmann's disease 
and Oppenheim's disease. Garrod, Batten, Thursfield and Paterson (1934) use the term infantile muscular atrophy of spinal origin to cover all types of case of this kind.

From a consideration of the findings in many cases described under the headings Werdnig-Hoffmann's disease and Oppenheim's disease, the following broad outline of the outstanding features of infantile muscular atrophy of spinal origin may be given:

(a) The disease is present at birth or develops gradually during the early months of life.

(b) A familial incidence is common.

(c) The sexes are equally affected.

(d) Muscle wasting may be generalized or may be more prominent in certain areas, particularly in the trunk, neck and proximal limb segments. It is often obscured by a thick layer of subcutaneous fat.

(e) The muscles are hypotonic, markedly so in some cases, and the tendon reflexes are lost.

(f) Paralysis of the intercostal muscles is common. Secondary results are a prominent abdomen, a weak cry, laboured coughing. and difficulty in sucking.

(g) Rarely cranial nerve palsies occur.

(h) Some cases develop contractures.

(i) Sensation and mentality are unimpaired in all cases.

(j) The prognosis is poor. The majority die in a few months from pneumonia. A small number, especially the congenital type with a good family history, and no local wasting, tend to improve.

(k) At autopsy the findings are atrophy of anterior horn cells, and secondary atrophy of muscle.

That the position may not be as simple as is indicated above is suggested by the cases presented by Turner (1940), which showed a gradual transition from a state of amyotonia with marked flaccidity and absence of local muscular atrophy to a state of myopathy with local muscular atrophy and absence of flaccidity. As a number of cases of Oppenheim's disease have been reported (Lereboullet and Baudouin, 1909; Councilman and Dunn, 1911: Haushalter, 1920; Silverberg, 1923; and Menges, 1931) in which no evidence of any spinal cord lesion was discovered in spite of careful search, Turner concludes that ' as was indicated by Spiller (1914), amyotonia congenita is a symptom rather than a disease, and it may be caused either by a congenital myopathy as in the family reported here, or by a spinal affection of unknown etiology which bears a close relation to Werdnig-Hoffmann's disease.'

If this conclusion is correct it is possible that the relatively few recorded cases which have improved considerably were myopathies rather than muscular atrophies. This is the type of case in which pathological confirmation will be difficult to obtain.

The etiology of muscular atrophy and of the myopathies is unknown.

Bicknell (1940) has reviewed recent work with vitamin $E$, which suggests that this factor may be important etiologically and therapeutically both in the 
muscular atrophies and in the myopathies. Ringsted (1935) and Einarson and Ringsted (1938) feeding adult rats on a diet free from vitamin $E$ found that there developed, after a period of fifteen weeks, ataxia of the hind legs which later became extreme. This was followed by flaccid paralysis and gross wasting. The condition of the rats was otherwise good. The pathological picture resembled a combination of tabes and progressive muscular atrophy, there being degeneration of the posterior roots, posterior columns and later of the anterior horn cells. Muscle degeneration was also present. Later work by Morgulis and Spencer (1936), Morgulis, Wilder and Eppstein (1938), Olcott (1938), and Madsen (1936), in addition to earlier work by Evans (1928), showed that this muscle degeneration may be a primary procedure in young animals. Regeneration of the wasted muscles occurred on addition of vitamin $E$ to the diet. Bicknell suggests that the myopathies and amyotrophic lateral sclerosis may be interpreted as the same deficiency disease, the former occurring in children and the latter in adults. The same author suggests that a dietetic insufficiency of vitamin $E$ may readily occur, and he claims good results in eighteen cases of muscular dystrophy, in four cases of amyotrophic lateral sclerosis, and in one case of amyotonia congenita treated with fresh dried whole wheat germ $\frac{1}{2}$ oz. twice daily. Bicknell states that it is important to realize that the anti-sterility factor-i.e. alpha tocopherol-is probably not identical with the myotrophic and neurotrophic factors (Goettsch and Ritzmann, 1939), though they all occur together in wheat germ oil. Wechsler (1940) also claims good results in cases of amyotrophic lateral sclerosis treated with vitamin $E$ together with the vitamin B complex.

These claims, however, have not been substantiated by other workers, notably Shelden et al. (1940), Denker and Scheinman (1941), and Ferrebee et al. (1941). The position is therefore still obscure, but at present it would appear that treatment of cases of infantile muscular atrophy of spinal origin with vitamin $E$ is well worth a trial. Should some of these cases, as Turner suggests, prove to be myopathies the same treatment would apply. It has been pointed out (Lancet, 1940, 1941) that as "the amount of creatinine in the urine (on a creatine-free diet) depends on the amount of improperly functioning muscle, its estimation provides a convenient biochemical method of assessing therapy.' It is obvious, also, that an insufficiency of vitamin $E$ in the maternal diet both in the ante-natal and in the post-natal periods is possibly an important factor in the etiology of infantile muscular atrophy of spinal origin.

\section{Case reports}

Case 1. G. S. Admitted aged 8 weeks.

History. Since birth the child had been unable to move any limb, apart from slight jerkings of the forearms and of the legs below the knees. The limbs were limp and held persistently in the same position, the arms being flexed across the chest, and the lower extremities extended. The child appeared to breathe with the abdomen only. There were no other complaints. The appetite was good. There was no difficulty in sucking or swallowing and no vomiting. The bowels were regular. There was no cough.

The mother's pregnancy was uneventful. Labour lasted two hours after the onset of severe pains. No instruments or anaesthetic were required. The child was temporarily cyanosed at birth. Breast feeding was not attempted because of difficulty with other children due to painful nipples and the infant was reared on a patent milk food with regular four-hourly feeds. No cod-liver oil or orange juice was given.

FAMILY HISTORY. Father, aged thirty-four years. Mother, aged twentysix years. Children: (1) M., aged seven years. (2) F., aged six years. 
(3) M., aged five years. (4) M., aged eight weeks, the patient. Parents and children were all alive and well. There were no miscarriages and no family history of paralysis or tuberculosis.

Examination. A poorly nourished, quiet baby. Weight $8 \mathrm{lb} .14 \mathrm{oz}$. Circumference of skull 15 in. Anterior fontanelle of normal tension and size. Right side of forehead more prominent than left. No palpable abnormality of limb bones or spine. Chest poorly formed, lower ribs in-drawn. Abdomen prominent. Skin of good colour and fine texture; warm and dry; no cyanosis or jaundice. Temperature $98 \cdot 4^{\circ} \mathrm{F}$. Pulse 150 per minute. Respiration 40 to 50 per minute.

LOCOMOTOR AND CENTRAL NERVOUS SYSTEM. No evidence of mental deficiency. Upper arm adducted, forearm flexed and pronated, hand flexed, fingers extended. Lower limbs extended, feet plantar flexed. The range of voluntary movement did not exceed $15^{\circ}$ at any joint, whereas a full range of passive movement was easily effected. Marked protrusion of abdomen on inspiration; screening showed normal diaphragmatic movements; costal movements not reported, but clinically respiration appeared to be entirely diaphragmatic. Muscles hypotonic; no obvious atrophy; no fibrillation seen. These last two features may have been obscured by the subcutaneous fat.

Tendon reflexes absent. No sensory impairment detected. Pupils dilated, equal, central, circular, reacting to light and accommodation. No ptosis, strabismus, or nystagmus. No facial weakness, and no difficulty in sucking or swallowing.

Cerebrospinal fluid: clear and not under pressure; nothing pathological in stained film; protein $20 \mathrm{mgm}$. per cent.; sugar $76 \mathrm{mgm}$. per cent.; chlorides $714 \mathrm{mgm}$. per cent.

Clinical examination of the other systems failed to reveal any abnormality. $\mathrm{x}$-ray of chest on admission showed both lungs normally aerated. There was no abnormality of cardio-vascular system.

Progress. One week after admission the respiration rate increased to about 80 per minute, and the temperature rose to $99 \cdot 5^{\circ} \mathrm{F}$. Feeding became very difficult owing to the dyspnoea. Physical signs of bronchopneumonia developed on the right side of the chest, and the child died five days later.

Report of necropsy. The body was that of a poorly nourished male infant aged two months, showing some deformity of the chest, with indrawing of the lower ribs. There was no rickety enlargement of the rib junctions or epiphyses. eye.

CENTRAL NERVOLS SYSTEM presented no abnormality visible to the unaided

RESPIRATORY SYSTEM. Both lungs were the seat of acute bronchitis and bronchopneumonia. Consolidation was extensively confluent in the right lung. The upper respiratory passages showed inflammatory changes. There was an early fibrinous pleurisy on the right side.

HeART was dilated on the right side.

Nothing of pathological interest was found in the other systems.

\section{Microscopical report}

SPINAL CORD. Sections from the cervical and lumbar enlargements were examined. Both showed identical changes. The number of motor nerve cells in the anterior horns of grey matter was greatly reduced. The remaining cells were much reduced in size, shrunken and altered in shape. The Nissl substance was decreased in amount and, in many cells, present only at the periphery. Many of the nuclei were pyknotic (fig. 1). A few of the nerve cells showed satellitosis, being surrounded by a group of small cells derived from oligodendroglia or microglia. This indicated a progressive degenerative process in the cells concerned, and proved that the changes were not the result 


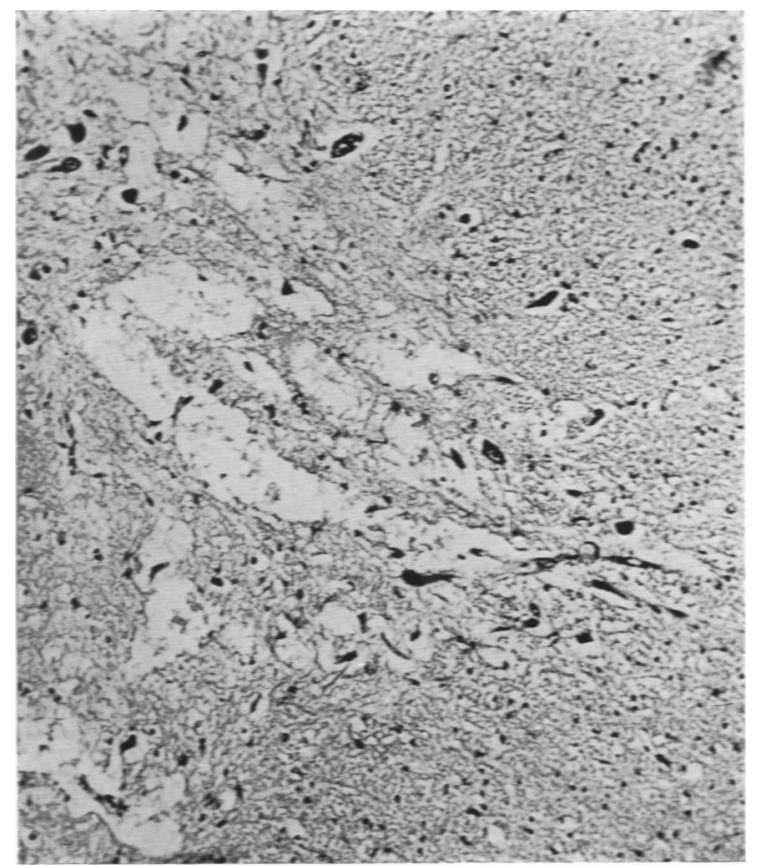

Fig. 1.-Case 1. Cervical cord, anterior horn, showing decreased number and shrunken pyknotic condition of motor nerve cells. $\mathrm{H}+\mathrm{E}: \times 100$.

of post-mortem autolysis. Sections stained by selective methods for the various tissue elements of the central nervous system were not available in this case, but there appeared to be a slight increase of glia fibres in the affected parts. There was no perivascular infiltration, hyperaemia, or other evidence of inflammation.

BraIN. In sections from the medulla oblongata, midbrain and cerebral cortex, no pathological changes were found.

Microscopical examination of the muscular tissues was not carried out.

Case 2. J. C. Admitted aged four weeks.

History. Complaint of weakness since birth and of 'turns' on several occasions. After birth it was noticed that the baby's arms were weak and that movements were limited to slight flexion at the elbows. The wrists were dropped. Legs were weak, but this was less obvious. From birth ' he has breathed with his stomach.' However, the family doctor states that flaccidity of limbs and head was noticeable at birth, but the respiratory difficulty was not so noticeable for some days. On one occasion nurse laid the baby on its abdomen, and respiration was so curtailed that the child became cyanosed. At age of one week the child 'took a turn' when he became limp and pale. Breathing was laboured. He did not twitch or become rigid, and he recovered in a few minutes. The mother thought he was becoming stronger until four days before admission when he 'took another turn.' This was more severe and lasted half an hour. He became limp and then cyanosed.

Feeding was by breast, supplemented. There was no difficulty in sucking or swallowing, no vomiting, the bowels were regular, but motions were green for a week before admission. There had been some recent loss in weight. There was no cough. 
The mother's pregnancy was normal. Labour, which occurred at full-time, lasted two days; "never had strong pains '; the head was on the perineum for a considerable time, and child easily delivered by forceps. Birth weight 7 to $8 \mathrm{lb}$.

FAMILY HISTORY. Father and mother both 28 years of age and in good health. No other children. No miscarriages. No family history of paralysis.

Examination. Length $22 \frac{1}{2}$ in. Head circumference $14 \frac{1}{2}$ in. Anterior fontanelle three finger-breadths open and of normal tension. No depression or other visible evidence of cranial damage. Chest poorly developed with upper ribs drawn in at the sides, and lower ribs splayed outwards. Spine and limbs of normal proportions and no evidence of any abnormality on clinical or radiological examination.

Body and limbs markedly thin; weight $6 \frac{3}{4} \mathrm{lb}$. Skin of normal colour and texture. No cyanosis or jaundice. Temperature $97 \cdot 2^{\circ} \mathrm{F}$. Pulse 130 per minute. Respiration $\mathbf{4 0}$ to $\mathbf{5 0}$ per minute.

LOCOMOTOR AND CENTRAL NERVOUS SYSTEM. Child lay in characteristic position with both arms flexed at elbows and the bilateral dropped wrists lying on chest. Knees slightly flexed. Both feet appeared to be dropped, but less obviously so than the wrists (fig. 2 and 3 ). All muscles poorly developed

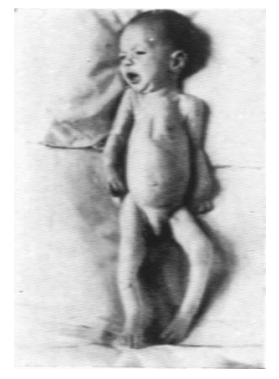

Fig. 2.-Case 2. J. C., aged 4 weeks.

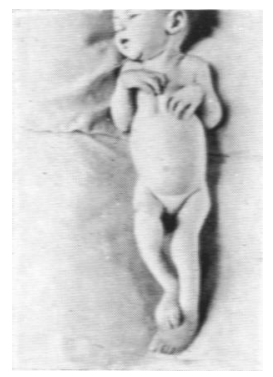

FIG. 3.-Case 2. J. C., aged 4 weeks.

and of poor tone. Flexion of elbows easily overcome. Slight voluntary movement of flexion at elbows, and a suspicion of movement at shoulders. Poor movement of fingers, but no power of extension of wrists. Slight flexion of knee, and even less power of extension. Slight movement of toes. Diaphragmatic respiration with indrawing of intercostal muscles and marked protrusion of abdomen on inspiration. Abdominal muscles thin and of poor tone. No tendon reflexes elicited. No muscular twitching or fibrillation visible. Sensation to pin-prick appeared intact as child cried and attempted to withdraw limb. Cry very feeble. No ptosis or strabismus. Eye movements normal. Pupils circular, average size, equal and both reacting to light. No facial weakness. Sucking and swallowing unimpaired.

Lumbar puncture gave a drop of gelatinous fluid, followed by clear fluid with a faint yellowish colour. No evidence of increase in pressure. Pathologists report: 'Fluid contained small flakes which consisted of groups or sheets of cells with round nucleus, and a fairly large body, possibly cells of endothelial type. No organism found. Culture sterile.' Biochemistry: protein $22 \mathrm{mgm}$. per cent.: chlorides $722 \mathrm{mgm}$. per cent.: sugar $81 \mathrm{mgm}$. per cent.

Clinical examination of the other systems failed to reveal any abnormality. X-ray of chest: 'abnormal contour right cardiac border: ? congenital heart lesion.' Haemoglobin 87 per cent.; white blood cells 14,800 per c.mm. 
Progress. Child's condition remained stationary for several days, but then feeding became difficult and progressive weakness terminated in death one week after admission. Temperature in ward $97.8^{\circ}$ to $98.6^{\circ} \mathrm{F}$.

Report of necropsy. The body was that of a poorly nourished male infant, aged five weeks, showing deformity of chest, with forward projection of the sternum and indrawing of the ribs at the sides. The abdomen was distended and the anterior abdominal wall was very thin.

CENTRAL NERVOUS SYSTEM. Brain and spinal cord presented no abnormality visible to the unaided eye.

RESPIRATORY SYSTEM. There was extensive atelectasis of the lungs, especially the right, a large part of which was not aerated. There was no pneumonia. The upper respiratory passages were healthy.

HEART was a little dilated on the right side.

Nothing of pathological interest was found in the other internal organs.

\section{Microscopical report}

SPINAL CORD. Sections from various levels showed the same changes as those found in case 1. There was a similar reduction in the number and size of the motor nerve cells in the anterior horns, with the same changes in the structure of the cells (fig. 4). Here again a few of the cells showed satellitosis

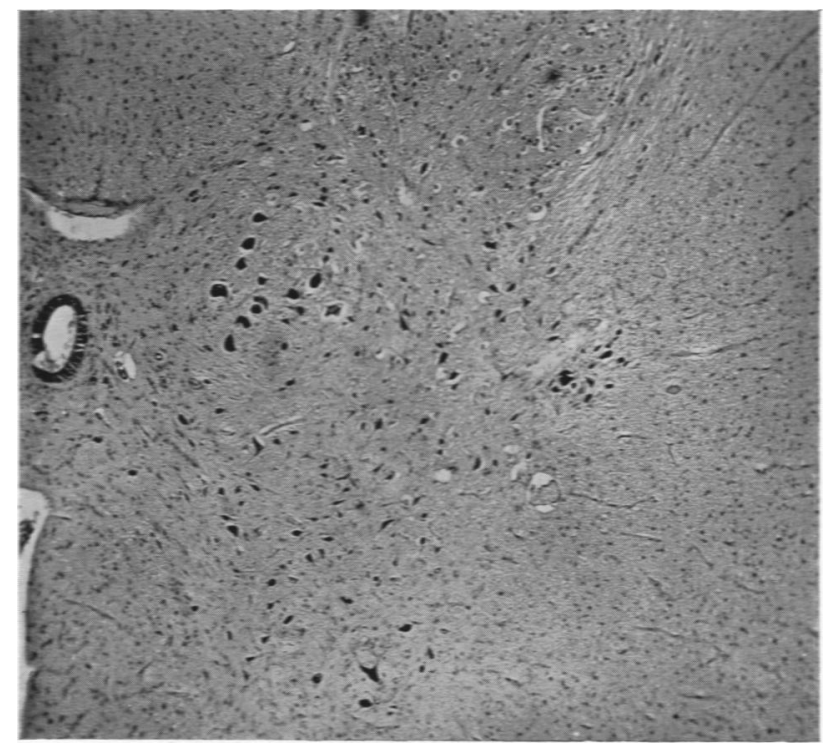

Fig. 4.-Case 2. Dorsal cord, showing anterior and posterior horns; decreased number and size of anterior horn cells; posterior horn cells unaffected. Iron haematoxylin; $\times 50$.

(fig. 5). Sections stained by Anderson's Victoria blue method for neurolgia fibres showed a slight but definite gliosis in the region of the anterior horns, with increase of both fibres and nuclei. Sections stained with Scharlach R. revealed no fat in stainable form, indicating that no rapid degeneration of myelin was in progress. Sections of the cauda equina stained by Spielmeyer's method for myelin sheaths showed pronounced pallor of the anterior nerve roots, indicating a great scarcity of myelinated fibres there, while the posterior nerve roots were well myelinated (fig. 6). 
BRAIN. Sections from the medulla oblongata, pons, midbrain, and various parts of the cerebrum showed no pathological changes.

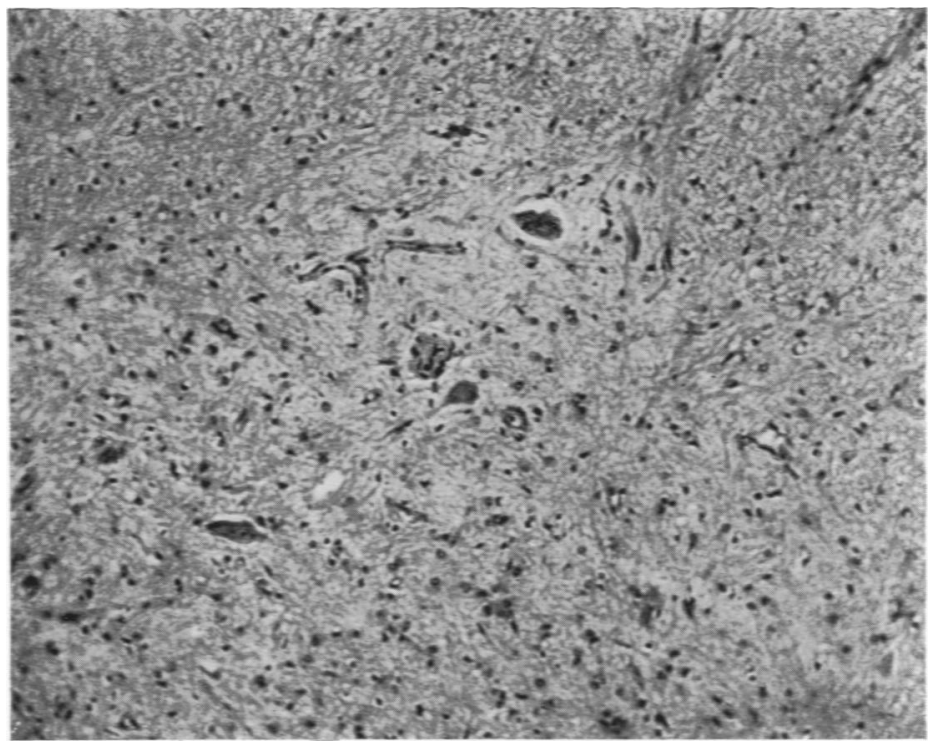

FIG. 5.-Case 2. Anterior horn, showing reduction of number of motor nerve cells, and degeneration of remaining cells. One cell in centre of field shows satellitosis.

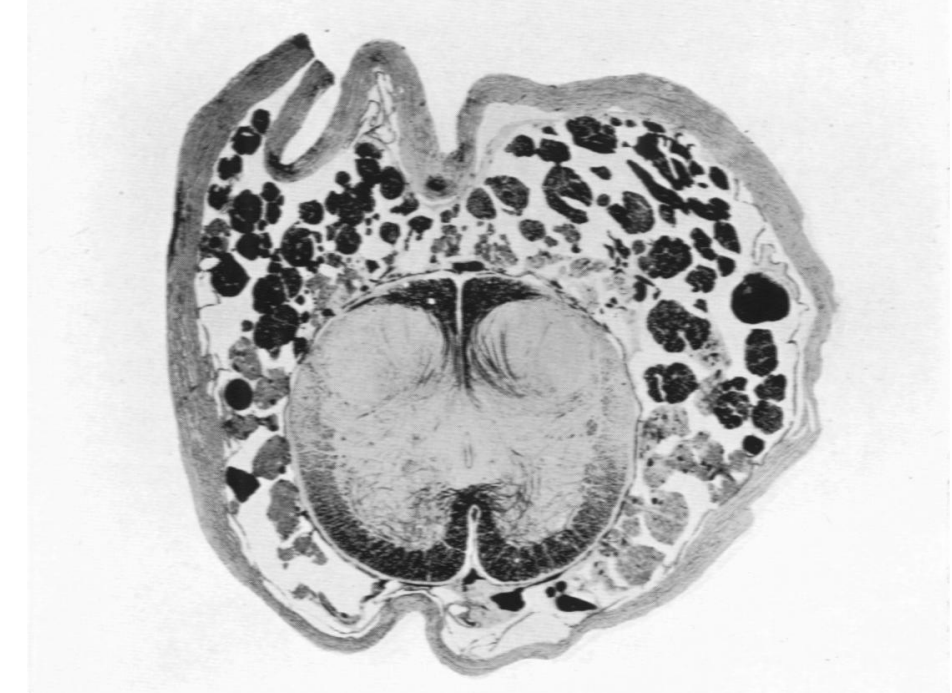

Fig. 6.-Case 2. Cauda equina showing degeneration of anterior nerve roots. Weigert Pal: $\times 10$.

MuSCLES. Sections from heart muscle, urinary bladder, and a selection of voluntary muscles were examined. The heart and urinary bladder showed 


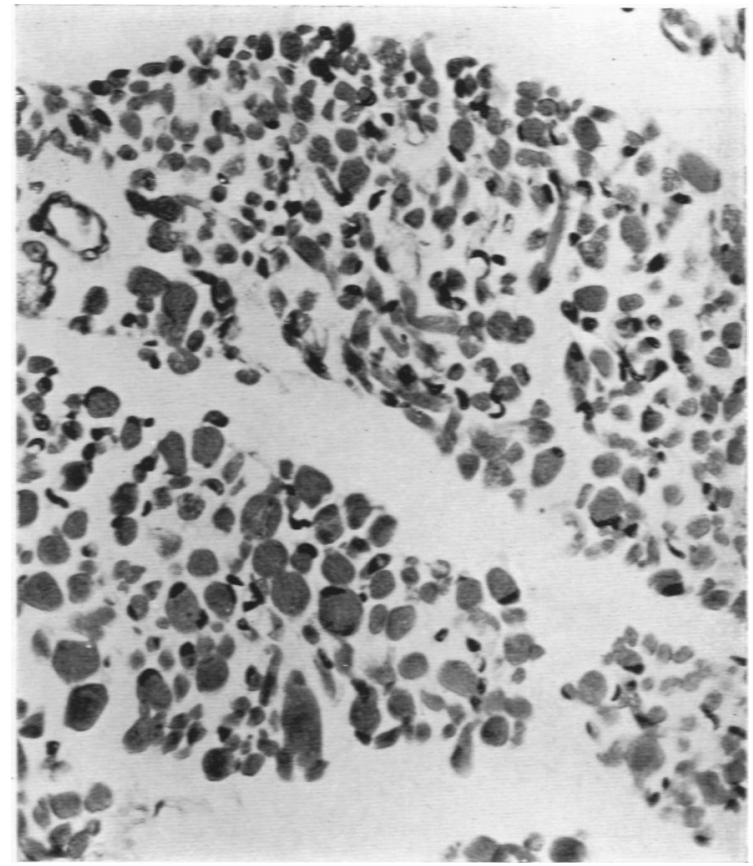

Fig. 7.-Case 2. Psoas muscle, showing detail of changes in muscle fibres. $H+E ; \times 400$.

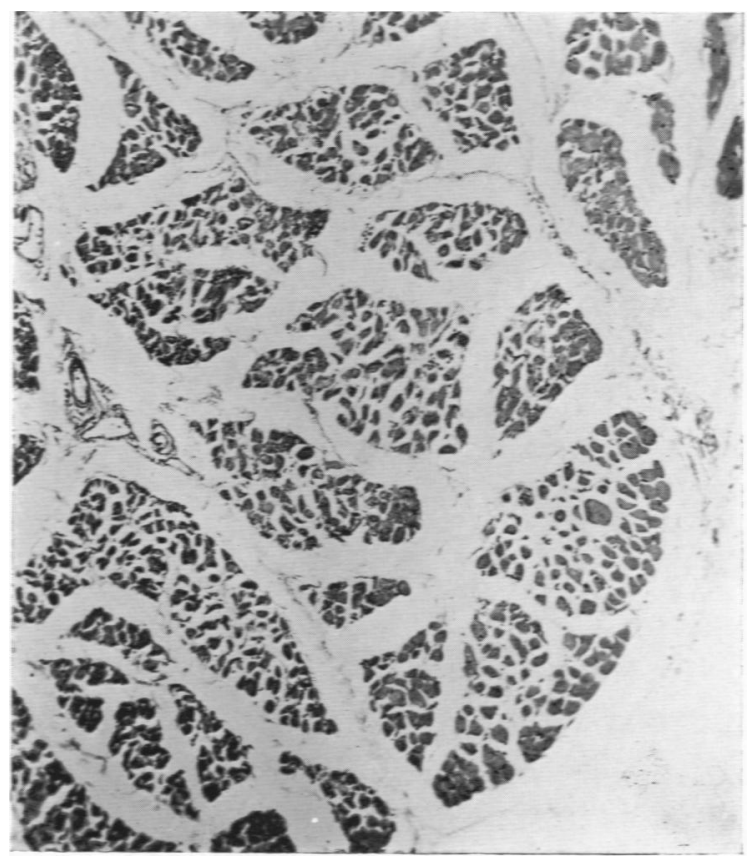

FIG. 8. - Case 2. Diaphragm, showing atrophy of muscle fibres, with irregularity of size. $\mathrm{H}+\mathrm{E} ; \times 8 \mathbf{8 0}$. 
nothing abnormal. The voluntary muscles all showed pathological changes. Many of the muscle fibres were greatly atrophied. The atrophy was variable, so there was a striking irregularity in the size of the fibres, even within a single bundle, some being of almost normal size whilst others were reduced to mere threads (fig. 7). Some of the most atrophied fibres were fragmented. Crossstriation was retained in the larger fibres, but could not be detected in many of the smaller. No change resembling Zenker's necrosis was observed. The fibrous stroma of the muscles was not increased. In the case of the diaphragm, the whole thickness of the muscle was much decreased, in comparison with that of a normal infant of the same age, and the same atrophy of fibres, with irregularity of size, was found as in the other skeletal muscles (fig. 8).

\section{Discussion}

The findings in both cases are in keeping with those outlined above as typical of infantile muscular atrophy of spinal origin. Vitamin $E$ was not given a trial in either case. The atrophic changes noted in the diaphragm in case 2 have not previously been described in any recorded case that has come under our notice. Several authors, including Greenfield and Stern (1927), and Paterson (1928), have stated that the diaphragm is constantly spared. There is no obvious reason why this should be so, and it is all the more remarkable when, as was pointed out by Greenfield and Stern (1927), the frequency of severe involvement of the muscles of the neck at an early stage is considered. The evidence, however, in support of the diaphragm being unaffected is inadequate, as histological confirmation was obtained in only a minority of cases. Greenfield and Stern (1927) describe four cases of their own in two of which the diaphragm was examined histologically. The fibres were described as being small ( $15 \mu$ to $20 \mu$ in diameter), but as being uniform in size and shape. These changes were regarded as being normal for this muscle, and the authors conclude that 'the diaphragm . . . in all cases appeared normal.' Paterson (1928) records histological confirmation of a normal diaphragm in only one of the six cases described. He concludes, apparently mainly from clinical evidence, that, 'the intercostal and abdominal muscles are frequently affected, whereas the diaphragm remains unaffected.'

In case 2 respiration was undoubtedly diaphragmatic, and the explanation of this is probably that the changes in the intercostal muscles were in advance of those in the diaphragm. Definite atrophic changes may therefore be present in the diaphragm even if clinically it appears unaffected and indeed overacting.

\section{Summary}

The generally accepted conception of muscular atrophy in infants is outlined.

The comprehensive term infantile muscular atrophy of spinal origin is used rather than the terms Werdnig-Hoffmann's disease and Oppenheim's disease. Reasons for this choice are given.

The outstanding features of this disease are outlined.

The possibility that some cases may prove to be muscular dystrophy is mentioned. 
Attention is drawn to recent work with vitamin $E$, and to the possible importance of this vitamin etiologically and therapeutically in cases of infantile muscular atrophy of spinal origin.

Two new cases are described in detail and are discussed briefly, attention being drawn to the atrophic changes found in the diaphragm in one of these cases, a feature not previously recorded in the literature.

Thanks are due to Professor C. McNeil, and to the late Dr. J. McNair Murray for permission to publish these cases, and also to Dr. William Blackwood for two of the photomicrographs, to Dr. John Thomson for the photographs of the second case, and to Dr. J. L. Henderson for much helpful criticism. Dr. Agnes R. Macgregor kindly supplied all the pathological reports, and gave great help throughout.

\section{REFERENCES}

Bicknell, F. (1940). Lancet, 1, 10.

Councilman, T., and Dunn, C. H. (1911). Amer. J. Dis. Child., 2, 34J.

Denker, P. G., and Scheinman, L. (1941). J. Amer. med. Ass., 116, 1893.

Einarson, L., and Ringsted, A. (1938). Effect of chronic vitamin E deficiency on the nervous sistem and skeletal musculature in adult rats, Copenhagen.

Evans, H. M., and Burr, G. O. (1928). J. biol. Chem., 76, 273.

Ferrebee, J. W., Klingman, W. O., and Frantz, A. M. (1941). J. Amer. med. Ass., 116, 1895.

Garrod, Sir A. E., Batten, F. E., Thursfield, H., and Paterson, D. (1934). Diseases of children, London, third edition, p. 801.

Goettsch, M., and Ritzmann, J. (1939). J. Nutrit., 17, 371.

Greenfield, J. G., and Stern, R. O. (1927). Brain, 50, 652.

Haushalter, P. (1920). Arch. Méd. Enf., 23, 133.

Hoffmann, J. (1893). Dtsch. Z. Nervenheilk, 3, 427.

Huenekens, E. J., and Bell, E. T. (1920). Amer. J. Dis. Child., 20, 496.

Lancet, (1940), 2, 199, and (1941), 2, 220.

Lereboullet, P., and Baudouin, A. (1909). Bull. Soc. méd. Hóp. Paris, 27, 1162.

Madsen, L. L. (1936). J. Nutrit., 11, 471.

Menges, O. (1931). Dtsch. Z. Nervenkeilk., 121, 240.

Morgulis, S., and Spencer, H. C. (1936). J. Nutrit., 11, 573.

—, S.. Wilder, V. M., and Eppstein, S. H. (1938). Ibid., 16, 219.

Olcott, H. S. (1938). Ibid., 15, 221.

Oppenheim, H. (1900). Mschr. Psychiat. Neurol., 8, 232.

Paterson, D. (1928). Westminster Hosp. Rep., 20, 43.

Ringsted, A. (1935). Biochem. J., 29, 3, 788.

Shelden, C. H., Butt, H. R., and Woltman, H. W. (1940). Proc. Mayo Clin., 15, 577.

Spiller, W. G. (1904-5). Univ. Pa. med. Bull., 17, 342.

(1914). Brain, 36, 75.

Silverberg, M. (1923). Virchon's Arch., 142, 165.

Turner, A. (1940). Brain, 43, 163.

Tuthill, C. R., and Levy, M. G. (1931). Amer. J. Dis. Child., 41, 591.

Wechsler, I. S. (1940). J. Amer. med. Ass., 114, 948.

Werdnig, G. (1891). Arch. Psychiat., 22, 437. 